D) Check for updates

Cite this: Analyst, 2021, 146, 1705

\title{
Mineralization of dental tissues and caries lesions detailed with Raman microspectroscopic imaging $\dagger$
}

\author{
Shuvashis Das Gupta, (ID $\ddagger^{\mathrm{a}}$ Markus Killenberger, $\ddagger^{\mathrm{b}}$ Tarja Tanner, (D) b,d \\ Lassi Rieppo, (D) ${ }^{a}$ Simo Saarakkala, (D) a,c Jarkko Heikkilä, ${ }^{b}$ Vuokko Anttonen (D) ${ }^{\text {b,d }}$ and \\ Mikko A. J. Finnilä (D)*a
}

\begin{abstract}
Dental caries is the most common oral disease that causes demineralization of the enamel and later of the dentin. Depth-wise assessment of the demineralization process could be used to help in treatment planning. In this study, we aimed to provide baseline information for the development of a Raman probe by characterizing the mineral composition of the dental tissues from large composition maps $\left(6 \times 3 \mathrm{~mm}^{2}\right.$ with $15 \mu \mathrm{m}$ step size) using Raman microspectroscopy. Ten human wisdom teeth with different stages of dental caries lesions were examined. All of the teeth were cut in half at representative locations of the caries lesions and then imaged with a Raman imaging microscope. The pre-processed spectral maps were combined into a single data matrix, and the spectra of the enamel, dentin, and caries were identified by $K$-means cluster analysis. Our results showed that unsupervised identification of dental caries is possible with the $K$-means clustering. The compositional analysis revealed that the carious lesions are less mineralized than the healthy enamel, and when the lesions extend into the dentin, they are even less mineralized. Furthermore, there were more carbonate imperfections in the mineral crystal lattice of the caries tissues than in healthy tissues. Interestingly, we observed gradients in the sound enamel showing higher mineralization and greater mineral crystal perfection towards the tooth surface. To conclude, our results provide a baseline for the methodological development aimed at clinical diagnostics for the early detection of active caries lesions.
\end{abstract}

Received 28th September 2020
Accepted 12th November 2020

DOI: 10.1039/d0an01938k

rsc.li/analyst the plaque is below the critical value of $5.5 .^{2,3}$ Untreated dental caries can lead to dental, periapical, and even general infections and, consequently, is a major cause of tooth loss. ${ }^{4}$

The traditional treatment for cavitated caries lesions is to remove the infected enamel and dentin and place a filling. However, the prognosis is substantially better if caries lesions are detected at an early stage and their progression can be halted non-invasively. ${ }^{5}$ Caries is traditionally detected with a visual and tactile inspection aided by radiography (X-ray). ${ }^{6}$ Unfortunately, this system has a relatively low sensitivity yet high specificity. ${ }^{7}$ Conventional radiography tends to underestimate caries-induced tissue damage and demineralization. Additionally, it usually only detects lesions that have already extended beyond the enamel-dentin junction. ${ }^{8}$ Therefore, modern methods to detect early lesions are needed.

The healthy enamel is approximately 95\% mineral, $1 \%$ organic matter, and $4-5 \%$ water by weight. ${ }^{9}$ The mineral crystals in the enamel are arranged in a tightly packed rod-like pattern that forms the enamel prisms. Caries forms when the crystals begin to dissolve in the acidic environment of the dental biofilm. When the minerals are dissolved, the intercrystalline space increases, and the surface of the enamel becomes softer and more porous. ${ }^{3}$ The lesion will grow substantially 
quicker when it reaches the dentin as it is less mineralized. The pulp and dentin react to the stimuli from a caries lesion by sclerosis of the dentinal tubules and the formation of reactive dentin.

Raman spectroscopy uses monochromatic light (mostly in the near-infrared, visible, or UV range) that interacts with chemical bonds within the specimen. ${ }^{10}$ Most of the light is scattered elastically as Rayleigh scattering having the same wavelength as the original light. Some of the light (low probability: around 1 in $10^{8}$ photons), however, is scattered inelastically as Raman scattering with a different wavelength due to the change in the polarizability of molecules. The Raman scattering spectrum provides quantitative information of the molecular composition of the specimen. Raman spectroscopy is particularly well suited for studying biomaterials because of its high specificity for biomolecules and low interference with water. ${ }^{10}$ Moreover, it is a non-destructive technique and does not require molecular labeling.

Akkus et al. used Raman spectroscopy to study healthy enamel from extracted human incisors. ${ }^{11}$ They reported that the mineral content was lower in the cervical region than in the rest of the crown, and that the overall mineral content had substantial variance between individuals. Carious lesions have also been studied by Raman spectroscopy; ${ }^{12-16}$ the clinical and subclinical enamel lesions were found to have a lower mineral content in these studies. ${ }^{14,15}$ Wide-field Raman spectroscopy has also been used to determine regions of hypo- and hypermineralized lesions. ${ }^{17}$ Moreover, Raman polarization anisotropy has been used to detect early carious lesions. ${ }^{18}$ This method was based on the fact that there is a reduction in the polarization anisotropy of certain peaks in the Raman spectra from the caries lesion.

In our previous proof-of-concept study with a time-resolved complementary-metal-oxide-semiconductor (CMOS) singlephoton-avalanche-diode (SPAD) based Raman spectrometer, we have demonstrated that the fluorescence-suppressed Raman spectrometers have the potential to be used in clinical settings. ${ }^{19}$ Although time-resolved Raman spectroscopy holds great potential, detailed mapping has remained challenging. In continuation of these efforts, we aimed to use Raman microspectroscopy in this in vitro study to map the mineral composition of the dental tissues and caries lesions in a detailed manner. We hypothesized that enamel mineralization is not homogeneous and that a simple multivariate clustering technique can be used to detect even small carious lesions from the Raman maps.

\section{Experimental}

\section{Tooth samples}

The teeth in this study were extracted from patients in primary health care in the City of Oulu, Finland, who were being treated for dental caries, periodontitis or pericoronitis. In accordance with the Finnish law (Finlex chapter 6, section $20 \S$ (30.11.2012/689)), the extracted teeth were donated to the
University of Oulu, Research Unit of Oral Health Sciences, for research and education with the consent of the authorities of the City of Oulu. The identity of the patients cannot be tracked in any case. Ten third molar teeth were used in the study. The teeth had caries lesions of different stages. The samples (teeth) were disinfected by boiling and stored in 70\% ethanol. The caries lesions were examined visually with the International Caries Detection and Assessment System (ICDAS) grading system giving the samples a grade of lesion extension and activity. Radiographs (X-ray) were also acquired from the samples, and they were graded with the International Caries Classification and Management System (ICCMS). ${ }^{20}$ This was performed with a traditional clinical dental X-ray device (Kodak 2100 Intraoral X-Ray System, Eastman Kodak Company, USA). The clinical assessment data are shown in Table 1. The visual images and radiographs of two of the samples (one having a minor visible cavity and another with a large visible cavity) are shown in Fig. 1. Four samples had enamel caries lesions and six had dentinal caries lesions according to the clinical and radiographic diagnosis. Nine samples had active lesions and one had an inactive lesion. The radiographic assessment graded most of the samples at grade 3 , which indicates that the lesions had progressed to the outer $1 / 3$ rd of the dentin.

\section{Sample preparation}

The samples were prepared for Raman microspectroscopic measurements by removing the roots and cutting the samples in half at representative locations of the caries lesions with a low speed saw (IsoMet, Buehler, Illinois, USA). Next, the samples were cast in metallurgical resin (EpoThin 2, Buehler, Illinois, USA) for fixation. The samples' surfaces were finished with P600 abrasive paper for $3 \mathrm{~min}$, P1000 abrasive paper for $5 \mathrm{~min}$, and P2000 abrasive paper for $5 \mathrm{~min}$. Final polishing was performed with $6 \mu \mathrm{m}$ and $1 \mu \mathrm{m}$ MetaDi Supreme Polycrystalline Diamond Suspension, both for $15 \mathrm{~min}$, and

Table 1 Clinical assessment data from the samples including the ICDAS criteria and lesion activity, and the ICCMS radiographic grading. ICDAS grades 1 and 2 represent initial caries lesions, 3 and 4 moderate dentinal lesions, and 5 and 6 deep dentinal lesions; active lesion is labeled + and inactive lesion -. In ICCMS radiographic grading, 1 is an enamel lesion, 2 a deep enamel or superficial dentinal lesion, 3 a mid-dentinal lesion, and 4 a deep dentinal lesion

\begin{tabular}{lll}
\hline $\begin{array}{l}\text { Sample } \\
\text { number }\end{array}$ & $\begin{array}{l}\text { ICDAS and activity } \\
\text { grading }\end{array}$ & $\begin{array}{l}\text { ICCMS radiographic } \\
\text { grading }\end{array}$ \\
\hline 1 & $2+$ & 3 \\
2 & $3+$ & 3 \\
3 & $5+$ & 4 \\
4 & $1+$ & $3 / 2$ \\
5 & $5+$ & 3 \\
6 & $2+$ & 3 \\
7 & $3+$ & $2 / 3$ \\
8 & $6+$ & 3 \\
9 & $3+$ & 3 \\
10 & $2-$ & 3
\end{tabular}


With visibly minor cavity With visibly large cavity
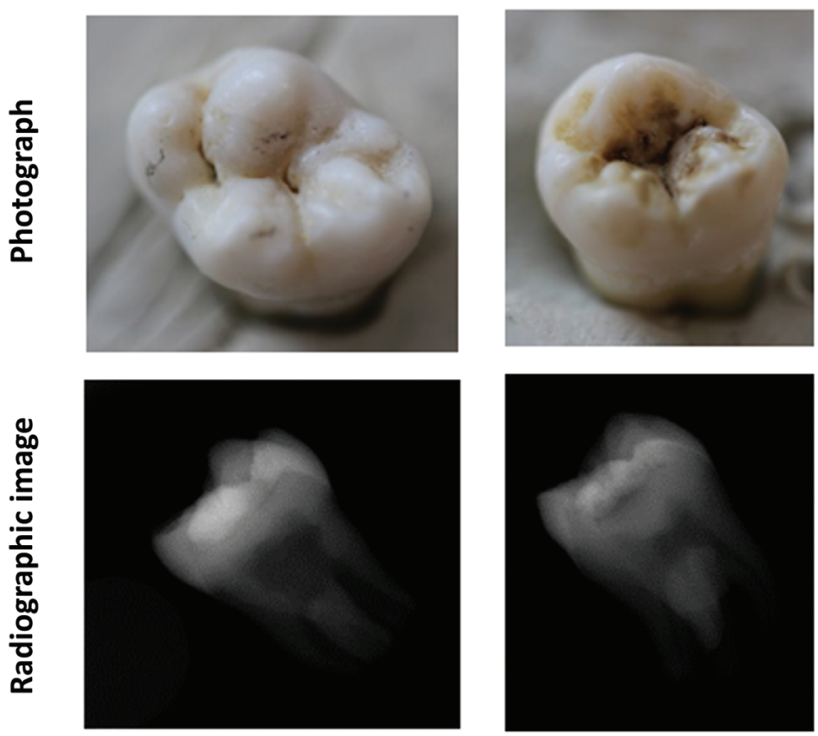

Fig. 1 Visual and radiographic images of the samples prior to the preparation. Although clinically, the left sample has an initial enamel caries lesion with minor cavitation, in the radiographic image, the lesion extends past the enamel-dentin junction slightly into the outer $1 / 3 \mathrm{rd}$ of the dentin. Thus, it has a 2+ ICDAS grade and a 3 ICCMS radiographic grade. The right sample has a clinically large lesion extending into the dentin. The radiographic image also confirms this with the lesion extending into the outer $1 / 3$ rd of the dentin. ICDAS grading: $5+$, ICCMS radiographic grading: 3 .

finally $0.3 \mu \mathrm{m}$ MicroPolish Alumina suspension for $5 \mathrm{~min}$ using a speed of $150 \mathrm{rpm}$ and a force of $15 \mathrm{~N}$.

\section{Raman microspectroscopy}

A confocal Raman imaging system (DXR ${ }^{\mathrm{TM}} 2 \mathrm{xi}$, Thermo Fisher Scientific, USA) equipped with a $10 \times / 0.3 \mathrm{NA}$ water immersion objective was used for acquiring the Raman hyperspectral maps. The samples were placed on a Petri dish and submerged in sterile-filtered de-ionized water for performing the measurements.

A $785 \mathrm{~nm}$ laser $(30 \mathrm{~mW})$ and a $50 \mu \mathrm{m}$ confocal pinhole aperture were used to excite the Raman scattering. The Raman spectra were collected for $0.025 \mathrm{~s}$ and averaged for 5 scans. A wide-range grating (spectral resolution of $5 \mathrm{~cm}^{-1}$ in the range of $50-3250 \mathrm{~cm}^{-1}$ ) was used for the Raman measurements. A rectangular region-of-interest containing the enamel, dentin, and caries lesions was selected for Raman mapping based on visual inspection of the full mosaic image (dark-field image using an optical microscope) for each sample. The measurement regions were mapped with a step size of $15 \mu \mathrm{m}$.

\section{Raman spectral pre-processing}

The raw spectra were truncated to the chemical fingerprint range (350-1750 $\left.\mathrm{cm}^{-1}\right)$ and subjected to a cosmic spike removal algorithm (sensitivity: 3, spikes width: 7). Subsequently, a principal component analysis-based noise filter with ten principal components (explained over $98 \%$ of variance) was applied to reduce the spectral noise. The baseline shifts due to tissue autofluorescence were corrected by fitting a twelve-point third-order polynomial to the spectra. Finally, the Raman spectra were vector-normalized to remove the differences in the total Raman intensity due to the physical and optical factors (i.e., Raman scattering efficiency, other optical effects, and surface roughness of the specimen). All pre-processing steps of the Raman spectra were performed using a commercial MATLAB toolbox (Cytospec 2.00.05, built 353, Berlin, Germany).

\section{Caries lesion identification ( $K$-means cluster analysis)}

Identification of the caries lesion along with the enamel and dentin tissues from the Raman maps was performed by $K$-means cluster analysis. In $K$-means clustering, the spectra are classified based on their (dis)similarity or "distance". It is an unsupervised technique and depends on the user-defined number of clusters.

The pre-processed Raman maps of all samples were combined into a single data matrix for the cluster analysis to reduce the sample-specific bias and increase the interpretability of the clusters. The number of clusters was increased iteratively, and after each iteration, a pseudo-colored cluster image was generated and "subjectively" matched with the microscopic images until the tissue-specific clusters matched the respective tissue locations from the microscopic images. After the final iteration, all clusters were annotated using the microscopic images as references. Subsequently, binary masks were generated for the enamel, dentin, and caries tissues by combining the clusters assigned to the same tissue type. Speckles were removed from the binary masks by performing areaopening operations (less than 3 pixels). Finally, the mean cluster spectra of each tissue type were determined.

\section{Chemical analysis}

The mineral composition of the dental tissues was investigated quantitatively by calculating the mineral-to-matrix ratio (the degree of mineralization), type-B carbonate substitution, and mineral crystallinity.

We used the $\mathrm{V}_{1} \mathrm{PO}_{4}{ }^{3-}$ band $\left(932-980 \mathrm{~cm}^{-1}\right)$ to represent the mineral content. ${ }^{17,21,22}$ This band is strongly polarization dependent, but as we used a depolarized laser for the excitation of the Raman scattering, we expected that this effect would not influence our measurements. The amide III band (1215-1300 $\mathrm{cm}^{-1}$ ) (less susceptible to the tissue orientation effects $^{23}$ ) was used to represent the organic matrix.

The type-B carbonate symmetric stretch band (1055-1090 $\mathrm{cm}^{-1}$ ) was normalized by the mineral band to measure the type-B carbonate substitution in the apatite lattice. ${ }^{24,25}$ Moreover, the full width at half maximum (FWHM) of the $\mathrm{V}_{1} \mathrm{PO}_{4}{ }^{3-}$ band was calculated, as it inversely correlates with the degree of mineral crystallinity (a measure of the mineral crystal size and/or perfection). ${ }^{22}$

Finally, 2-D chemical maps were constructed by calculating the ratios of the areas under the Raman bands (calculated for 
the local baseline within the wavenumber range) for each pixel.

\section{Statistical analysis}

The binary masks generated by cluster analysis were used to identify the pixels of the different tissue types from the Raman composition maps. The differences between the enamel, dentin, and caries in the distributions of the compositional parameters were analyzed by the Kruskal-Wallis test, the nonparametric equivalent of one-way analysis of variance (ANOVA) (R Studio, v1.3.959, RStudio, Inc.), which was followed by the Wilcoxon rank-sum test with Bonferroni corrections to identify the significance of the difference between two tissue types. In the statistical analysis of the mineral-to-matrix ratio, the pixels having undefined values (due to the lack of an organic signal, mostly in the enamel tissue) were ignored.

\section{Results}

\section{Caries lesion identification}

The unsupervised $K$-means cluster analysis of the combined data matrix is shown in Fig. 2. Using the microscopic images (Fig. 2A) as references, twenty-four clusters were found to be optimal to identify the spectra of the enamel, dentin, and caries tissues from the Raman maps. The pseudo-colored cluster images (Fig. 2B) were visually compared with the microscopic images to annotate the clusters for each tissue type. When compared to the microscopic images, ten out of the twenty-four clusters were annotated as background; nine clusters as caries; three clusters as enamel, and the remaining two clusters as dentin. Subsequently, these clusters were combined, and masks were generated for the enamel, dentin, and caries (Fig. 2C). The visual comparisons between the masks
(A)

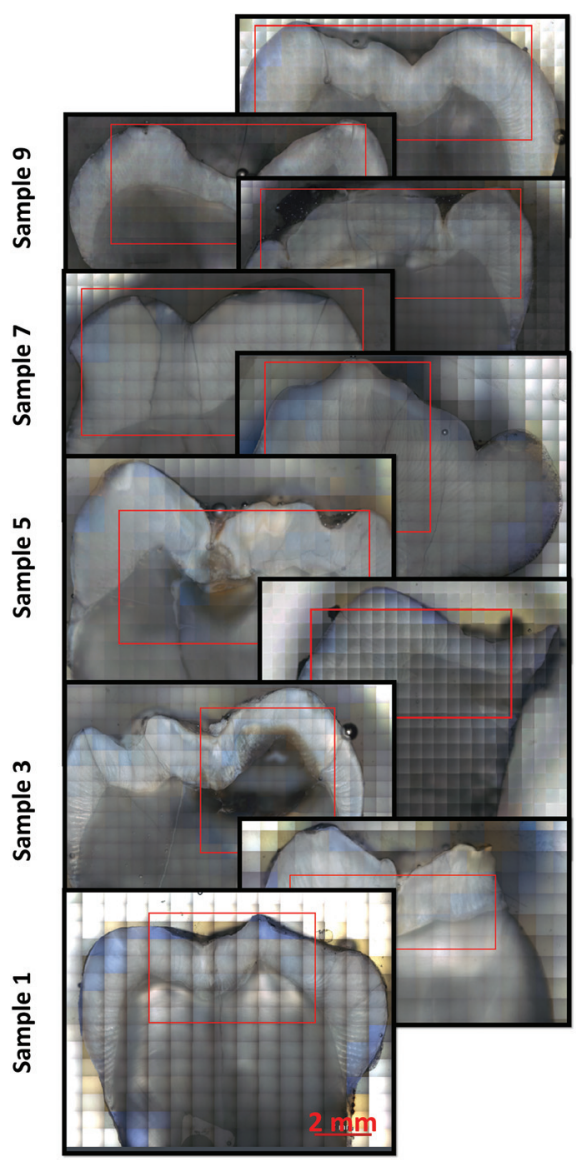

(B)

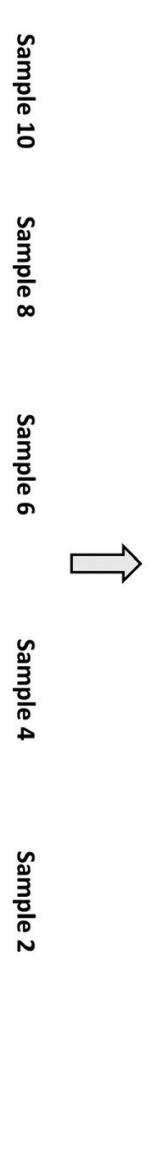

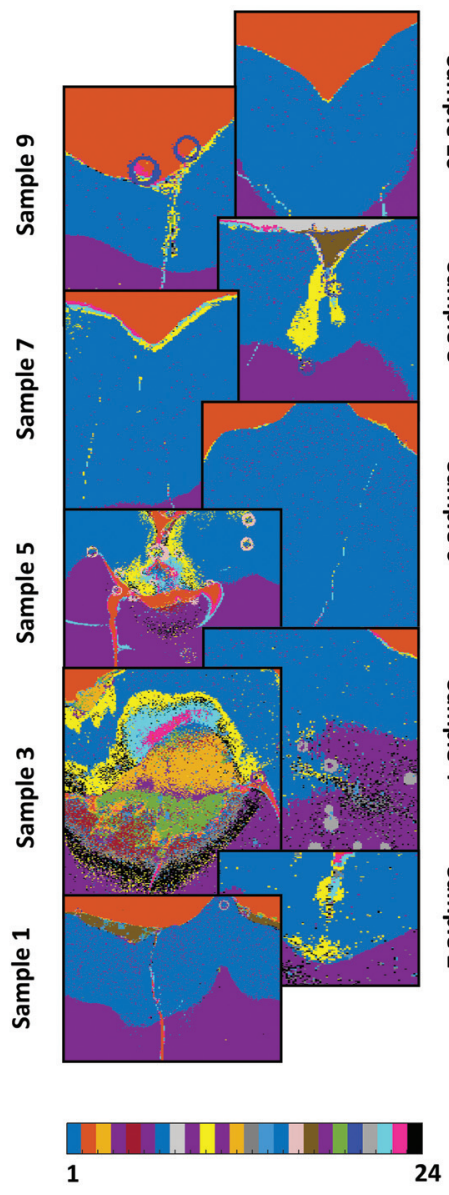

(C)

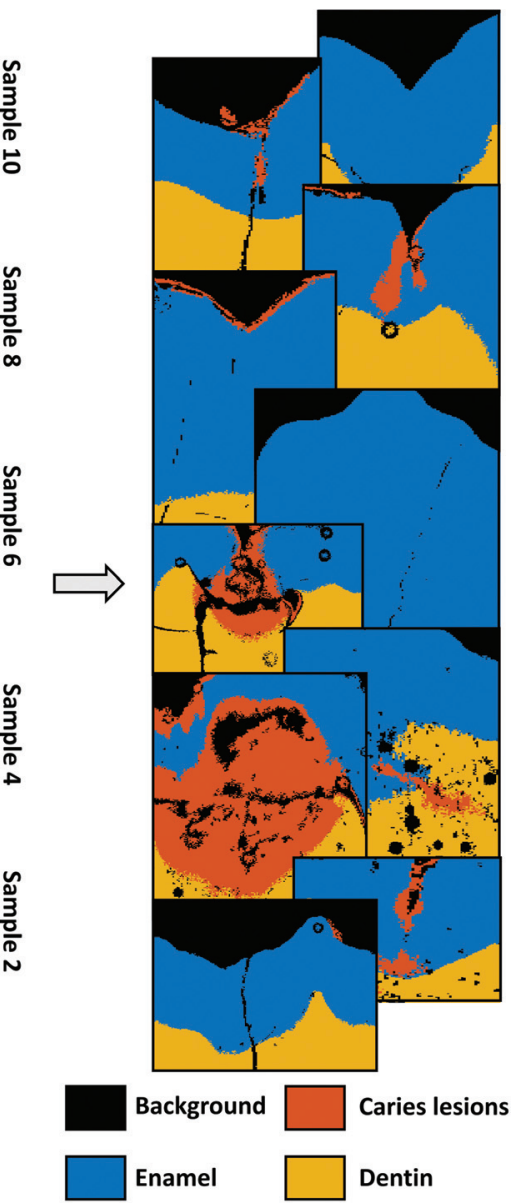

Fig. 2 Dental caries identification from Raman maps using $K$-means cluster analysis. (A) Microscopic images of ten human teeth. The red rectangular area represents the Raman measurement region. The regions-of-interest were further narrowed for the cluster and chemical analyses to reduce the computational load. (B) Pseudo-colored $K$-means cluster image of each sample. Twenty-four clusters were found to be optimal when the cluster analysis was performed on the combined data matrix. (C) False-color coded images of tissue-specific masks. Clusters representing the same tissue type were identified by comparing the microscopic images to the cluster images; then, tissue-specific clusters were combined to generate the masks. The masks were further subjected to despeckling to reduce noisy pixels. 
(A) Raw spectra (collected from a sample with large caries lesion)

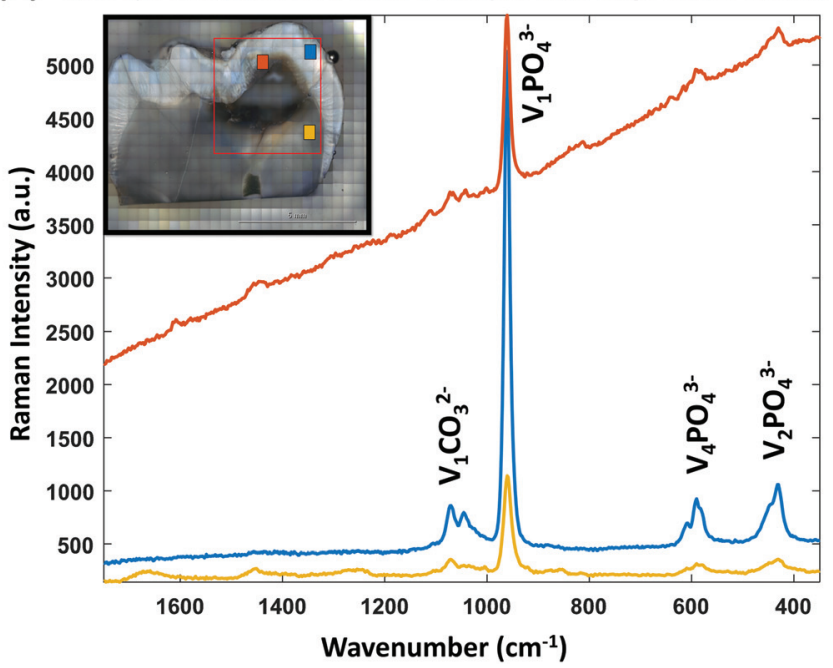

(B)

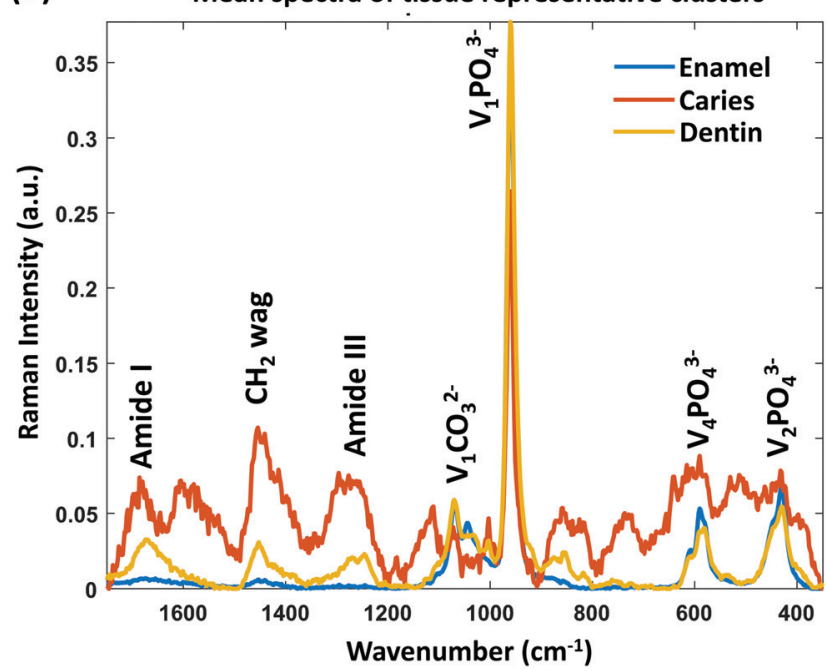

Fig. 3 (A) Raw Raman spectra of the enamel, dentin, and dental caries. The spectra were acquired by averaging forty spectra of each tissue from a sample with caries extended into the dentin. The measurement locations are color-coded on top of the microscopic image of the sample. The major mineral peaks are annotated. The raw spectra were then baseline-corrected, reproduced after principal component analysis with ten principal components, and vector-normalized. Subsequently, the spectra were subjected to $K$-means cluster analysis. (B) Mean spectra of tissue-specific clusters. The major mineral and organic peaks are annotated.

and macroscopic images indicate successful unsupervised identification of caries lesions from the Raman spectral maps.

The mean spectra of the clusters for the enamel, dentin, and caries are shown in Fig. 3B. The mean spectra of all twenty-four clusters are shown in ESI Fig. 1.† As expected, compared to the mean spectra of the dentin, the mean spectra of the enamel have higher values in the major mineral peaks and lower values in the organic peaks (ESI Fig. $2 \dagger$ ). Moreover, the mean cluster spectra of both enamel and dentin have higher values in the $\mathrm{V}_{1} \mathrm{PO}_{4}{ }^{3-}$ band compared to the mean spectra of the caries. Additionally, the raw Raman spectra (average of 40 spectra) of the enamel, dentin, and caries (approximate location) collected from a sample with caries lesions extended into the dentin are shown in Fig. 3A.

\section{Chemical analysis}

The chemical maps in Fig. 4B and D depict the degree of mineralization, mineral crystallinity, and type-B carbonate substitution in the samples with dentin caries and enamel caries (Fig. 4A and C), respectively. The cluster images of both samples are superimposed on the microscopic images.

The enamel lesions (Fig. 4C) were observed to be less mineralized and they have more substituted carbonate in the crystal lattice compared to the sound enamel (Fig. 4D). When the lesion extends into the dentin (Fig. 4A), the lesion tissues were observed to have even less mineral than the sound dentin, but many mineral apatites of caries were more crystalline and had less substituted carbonate in the lattice.

The chemical maps show that the sound (healthy) enamel tissues have a greater degree of mineralization and mineral crystallinity and less carbonate in the crystal lattice compared to the sound dentin. The mineral composition is not homo- geneous within the sound enamel tissue, but more crystallinity (greater mineral crystal size) and greater mineral crystal perfection are observed towards the tooth surface, whereas the mineral apatites are less crystalline and have more carbonate imperfection in the crystal lattice near the enamel-dentin junction. These gradients of the mineral crystal size and imperfection in the crystal lattice from the tooth surface to the junction of the sound enamel tissues are shown in ESI Fig. $3 . \dagger$ As there are many undefined mineral-to-matrix ratio values present in the sound enamel tissue, it is challenging to profile the gradient of the degree of mineralization. However, in Fig. 4D, a higher degree of mineralization can be observed towards the tooth surface.

In Fig. 5, the violin plots show the distributions of the Raman parameters in different tissue types for all samples, and the descriptive statistics (median and median absolute deviation) are presented in Table 2. The Kruskal-Wallis test shows that there is a statistically significant difference between the degree of mineralization of the different tissue types (chisquared value $=376554, p<0.001)$. The enamel has a higher $(p<0.001)$ degree of mineralization than the dentin and the caries lesions have a lower $(p<0.001)$ degree of mineralization than the enamel and dentin.

There is a statistically significant difference also between the mineral crystallinity of the different tissue types (chisquared value $=277515, p<0.001)$. The enamel is more crystalline than the dentin $(p<0.001)$. The caries lesions have higher crystallinity $(p<0.001)$ than the dentin but lower crystallinity than the enamel $(p<0.001)$.

Finally, the differences in the type-B carbonate substitution in the mineral crystal lattice between the different tissue types are statistically significant (chi-squared value $=264836, p<$ 
(A)

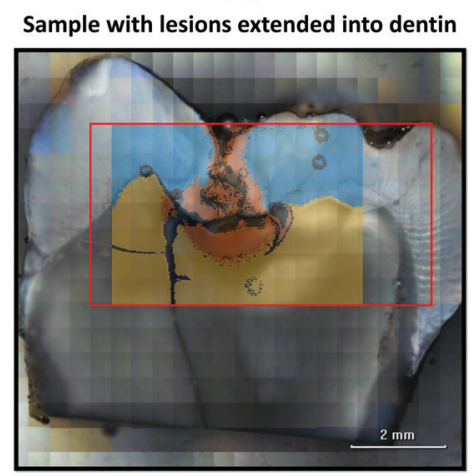

(B)
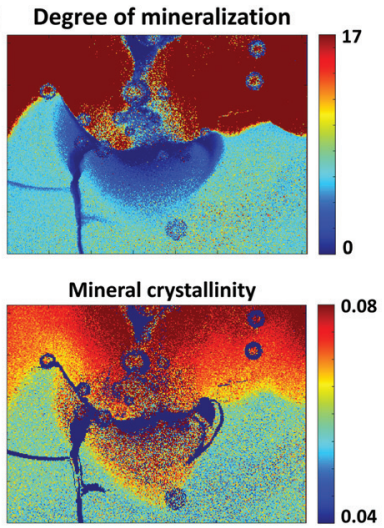

Type-B carbonate substitution

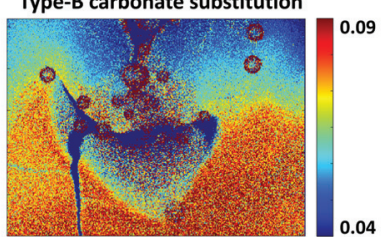

(D) Degree of mineralization

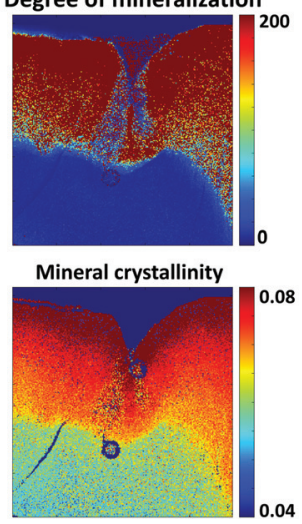

Type-B carbonate substitution

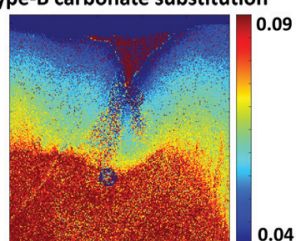

Fig. 4 (A and C) Microscopic images of samples having caries lesions extended into the dentin and having only enamel lesions, respectively. The cluster images are superimposed on top of the microscopic images. (B and D) The chemical maps of the mineral composition (degree of mineralization, mineral crystallinity, and type-B carbonate substitution) of both samples, respectively.
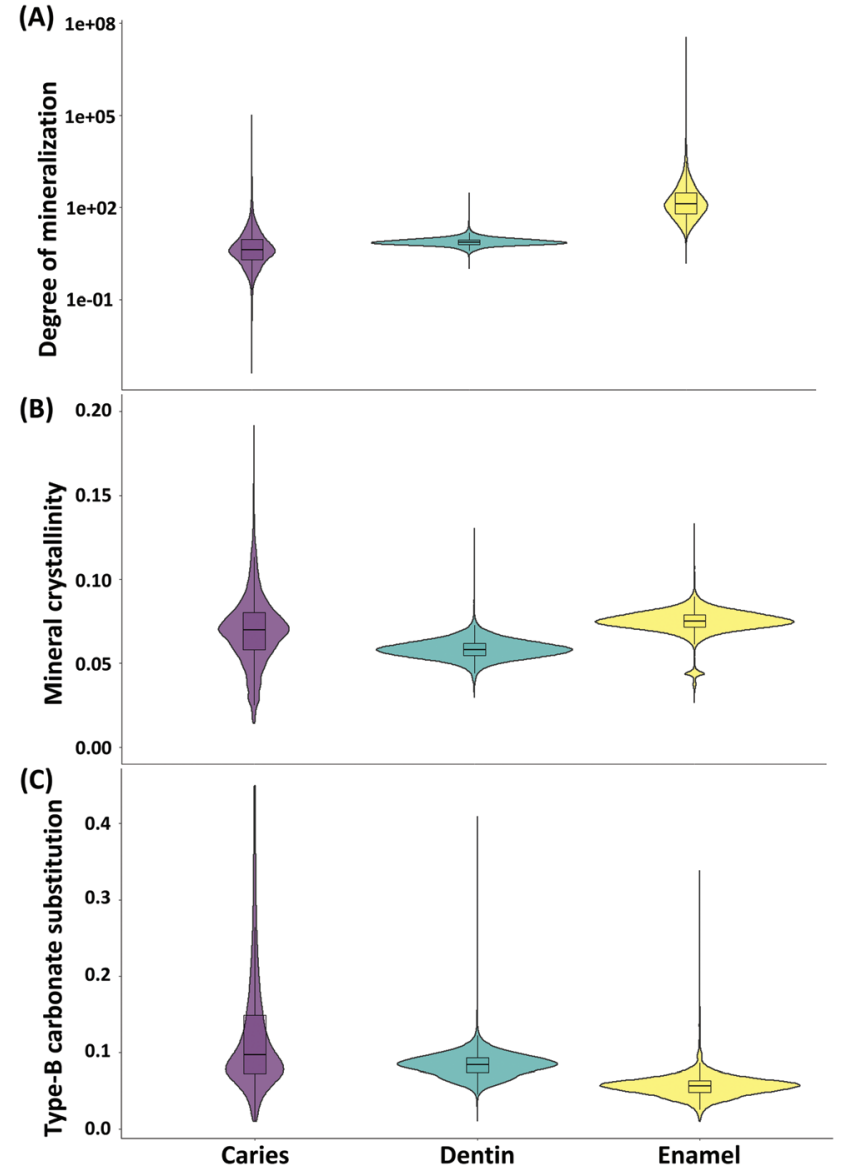

Fig. 5 The violin plots (including boxplots) showing the distributions of the (A) degree of mineralization, (B) mineral crystallinity, and (C) type-B carbonate substitution in the caries, dentin, and enamel.
Table 2 Descriptive statistics (median and median absolute deviation) of the distribution of the Raman parameters of the enamel, dentin, and caries tissues

\begin{tabular}{llll}
\hline Raman parameters & Tissue & Median & $\begin{array}{l}\text { Median absolute } \\
\text { deviation }\end{array}$ \\
\hline Degree of mineralization & Enamel & 130 & 127 \\
& Dentin & 7.32 & 1.81 \\
& Caries & 4.02 & 4.02 \\
Mineral crystallinity & Enamel & 0.0751 & 0.005 \\
& Dentin & 0.0581 & 0.00531 \\
Type-B carbonate substitution & Caries & 0.0697 & 0.016 \\
& Enamel & 0.0564 & 0.0113 \\
& Dentin & 0.0844 & 0.0142 \\
& Caries & 0.0965 & 0.0531
\end{tabular}

0.001). The enamel has a less $(p<0.001)$ substituted lattice compared to the dentin, indicating the presence of mineral crystals with greater stoichiometric perfection in the enamel than in the dentin. The caries lesions have a higher $(p<0.001)$ carbonate substitution in the lattice than the enamel and dentin, which indicates the presence of crystals with less stoichiometric perfection in the caries lesion.

\section{Discussion}

In this study, we spatially mapped the mineral composition of human teeth using Raman microspectroscopy. We identified caries lesions by $K$-means cluster analysis from the Raman spectral maps. This allowed us to investigate the quantitative changes in the mineral composition of dental hard tissues due 
to caries. The chemical analysis showed that carious lesions are indeed less mineralized than the enamel and even less mineralized when the lesion is extended into the dentin. Furthermore, the caries tissues had more stoichiometric imperfections in the mineral crystal lattice than the enamel and dentin. Finally, from the chemical maps, we observed a higher degree of mineralization with greater mineral crystallinity and lattice perfection towards the tooth surface in the sound enamel tissue. To our knowledge, we are the first to report these mineral composition gradients with composition maps in a detailed manner.

Previous studies have also used Raman spectroscopy to determine the mineral composition of dental hard tissues. ${ }^{14,15,17,26}$ Raman composition maps (using a step size of $50 \mu \mathrm{m}$ ) have been used previously to identify enamel demineralization caused by artificial caries using the intensity and FWHM of the $\mathrm{V}_{1} \mathrm{PO}_{4}{ }^{3-}$ band, depolarization ratio, and polarization anisotropy, ${ }^{26}$ where, based on the chemical analysis, it was concluded that demineralized enamel tissues have a disordered structure at very early stages.

Here, we applied $K$-means cluster analysis to identify dental caries from the Raman maps. $K$-Means cluster analysis is an unsupervised technique, but it depends on the user-defined number of clusters. We iteratively increased the number of clusters and visually compared the cluster images with the microscopic images after each iteration. Since we did not have the tissue-specific "true" labels for the microscopic images, objective validation of clustering could not be performed. To increase the interpretability of cluster analysis, we performed the clustering on the combined data matrix. This combined data matrix contains the Raman spectra of the enamel, dentin, caries with different severities, and resin, and other background noises due to aqueous measurement. This might be the reason why as many as twenty-four clusters were needed to segment all the tissue types. Overall, we observed that the cluster analysis was sensitive to the degree of mineralization. For instance, in the sample with severe caries lesions (sample 3, Fig. 2B), we found more than six clusters in the caries region. However, it should be possible to identify the different dental tissues with a smaller number of clusters if the clustering was performed on the Raman map of each sample individually. Furthermore, the limited sample set (10 samples) prevented us from associating specific clusters to caries progression.

The locations of the caries clusters were well correlated with the locations of the visually identified lesions (Fig. 2 and 4). The carious tissue clusters were located in the areas where caries are most typically found: either on the surface of the enamel or inside the enamel or dentin. In samples with severe clinical lesions, caries was also found grossly invading the dentin. Moreover, thin caries clusters were identified on the enamel surface in most of the samples that were assessed clinically as having active caries lesions. We suggest that those caries clusters represent the demineralization happening on the tooth surface. On tooth surfaces, there is a continuous demineralization-remineralization process going on. If the balance turns towards demineralization then sub-clinical and later clinically detectable lesions occur known as white spot lesions. Here, these surface clusters indicate that the oral circumstances of these samples having active caries lesions are favoring the demineralization process.

We found that the enamel carious lesions are less mineralized than the healthy enamel. This local demineralization might be due to the lactic acid produced by oral bacteria. When the demineralization process exceeds the remineralization process by saliva or clinical fluoride repair, the dental caries lesion originates at certain anatomical predilection sites on the teeth. ${ }^{5}$ We found that when the lesions extend into the dentin, they are even less mineralized than the dentin. Moreover, the values of all the analyzed Raman parameters are widely spread for the caries lesions. One of the reasons for this might be the combining of the values from the caries with different progression stages (surface caries, enamel caries, and dentin caries) in a single group.

In dental tissues, the type-B carbonate substitution happens when $\mathrm{CO}_{3}{ }^{2-}$ ions (impurity) occupy the $\mathrm{PO}_{4}{ }^{3-}$ sites of the hydroxyapatite crystal lattice. Carbonated hydroxyapatite is less stable, has lower hardness, and is more acid-soluble than non-carbonated hydroxyapatite. ${ }^{3,27}$ Here, caries lesions were found to be rich in substituted carbonate, and, therefore, they have stoichiometrically imperfect crystals. Stoichiometrically imperfect crystals are common in the demineralization process. Precipitations with high amounts of carbonate content are associated with caries lesions. ${ }^{28,29}$ Based on our mineral crystallinity results, these crystals of the caries tissues were thicker than those of the dentin tissues but thinner than the crystals of the enamel tissues (Table 2).

Previously, Fourier transform Raman microspectroscopic mapping was used to examine the distribution of phosphate (960 $\left.\mathrm{cm}^{-1}\right)$, carbonate $\left(1070 \mathrm{~cm}^{-1}\right)$, and $\mathrm{C}-\mathrm{H}$ stretch bands $\left(2700-2880 \mathrm{~cm}^{-1}\right)$ in a cross-section of a human tooth. ${ }^{30} \mathrm{An}$ increase of carbonate ions from the outside of the enamel towards the enamel-dentin junction was reported, while the phosphate ions showed the opposite gradient. Calcium hydroxyapatite and magnesium phosphate are the main sources of calcium and magnesium, respectively, in tooth enamel. In a recent study, ${ }^{31}$ an increase of calcium and magnesium contents towards the enamel-dentin junction in the central upper incisor teeth was reported using atomic absorption spectrometry. However, a decrease of the calcium content from the enamel surface towards the dentin has been also suggested. ${ }^{32,33}$ As magnesium ions act as a mineral growth inhibitor in calcium and phosphorus solutions, ${ }^{31}$ it is reasonable to assume that the magnesium content would increase with the decrease of the degree of mineralization towards the enamel-dentin junction.

This study used a relatively small laser step size $(15 \mu \mathrm{m})$ for the measurements, which gave a detailed spatial picture of the chemical properties of hydroxyapatite and allowed us to visualize the mineral composition gradient in the sound enamel. We observed gradients of decreasing degree of mineralization, mineral crystallinity, and lattice perfection (Fig. 4 and ESI 
Fig. $3 \dagger)$ from the occlusal surface towards the enamel-dentin junction. It could be that the dentin integrates into the enamel more coronally than towards the enamel-dentin junction or that the enamel contains more impurities as it approaches the dentin. Since the previous literature on enamel mineralization is contradictory, our results provide the much-needed validity to these observations.

In our proof-of-concept study, we imaged human teeth using a time-resolved CMOS SPAD-based Raman spectrometer and analyzed the Raman chemical image maps (step size: $250 \mu \mathrm{m}) .{ }^{19}$ A fluorescence suppressed time-resolved Raman spectrometer can be used to rapidly acquire comparable spectra with continuous wave Raman spectroscopy. Furthermore, a time-resolved CMOS SPAD-based Raman spectrometer can be used to collect depth-resolved chemical information. ${ }^{34}$ Our current study shows a natural decline in enamel mineralization which is less severe as in caries-associated demineralization. In continuation of the proof-of-concept study, the findings of this study provide baseline characteristics of caries lesions and depth-wise mineralization to enable the methodological development using a time-resolved Raman spectrometer aimed at clinical diagnostics for the early detection of active caries lesions.

\section{Conclusions}

To provide baseline information of teeth mineralization for the development of a Raman probe, we evaluated detailed mineral composition maps of human teeth with caries lesions imaged with Raman microspectroscopy. The dental caries lesions were identified from the spectral maps by applying $K$-means cluster analysis to the Raman spectra. The caries tissues had a lower degree of mineralization and more stoichiometric imperfections in the mineral crystal lattice than the enamel and dentin. In the sound enamel tissue, gradients of the degree of mineralization, mineral crystallinity, and lattice perfection were observed.

\section{Conflicts of interest}

There are no conflicts to declare.

\section{Acknowledgements}

This work was supported by funding from the European Union's Horizon 2020 research and innovation program under the Marie Skłodowska-Curie grant agreement no. 713645.

\section{References}

1 N. J. Kassebaum, E. Bernabé, M. Dahiya, B. Bhandari, C. J. L. Murray and W. Marcenes, J. Dent. Res., 2015, 94, 650-658.
2 R. J. Lamont and P. G. Egland, Molecular Medical Microbiology, 2nd edn, 2015, vol. 2-3, pp. 945-955.

3 C. Robinson, R. C. Shore, S. J. Brookes, S. Strafford, S. R. Wood and J. Kirkham, Crit. Rev. Oral Biol. Med., 2000, 11, 481-495.

4 M. A. Peres, L. M. D. Macpherson, R. J. Weyant, B. Daly, R. Venturelli, M. R. Mathur, S. Listl, R. K. Celeste, C. C. Guarnizo-Herreño, C. Kearns, H. Benzian, P. Allison and R. G. Watt, Lancet, 2019, 394, 249-260.

5 N. B. Pitts, D. T. Zero, P. D. Marsh, K. Ekstrand, J. A. Weintraub, F. Ramos-Gomez, J. Tagami, S. Twetman, G. Tsakos and A. Ismail, Nat. Rev. Dis. Primers, 2017, 3(1), 17030.

6 R. Macey, T. Walsh, P. Riley, A. M. Glenny, H. V. Worthington, J. E. Clarkson and D. Ricketts, Cochrane Database Syst. Rev., 2018, DOI: 10.1002/14651858.CD013215.

7 A. I. Ismail, J. Dent. Educ., 2004, 83, 56-66.

8 K. Bücher, M. Galler, M. Seitz, R. Hickel, K. H. Kunzelmann and J. Kühnisch, Oper. Dent., 2015, 40, 255-262.

9 M. Baldassarri, H. C. Margolis and E. Beniash, J. Dent. Res., 2008, 645-649.

10 H. J. Butler, L. Ashton, B. Bird, G. Cinque, K. Curtis, J. Dorney, K. Esmonde-White, N. J. Fullwood, B. Gardner, P. L. Martin-Hirsch, M. J. Walsh, M. R. McAinsh, N. Stone and F. L. Martin, Nat. Protoc., 2016, 11, 664-687.

11 A. Akkus, A. Akkus, R. Roperto, O. Akkus, T. Porto, S. Teich and L. Lang, J. Clin. Exp. Dent., 2016, 8, e546-e549.

12 A. C.-T. Ko, L.-P. Choo-Smith, M. Hewko, L. Leonardi, M. G. Sowa, C. C. S. Dong, P. Williams and B. Cleghorn, J. Biomed. Opt., 2005, 10, 031118.

13 Y. Wang, P. Spencer and M. P. Walker, J. Biomed. Mater. Res., Part A, 2007, 81, 279-286.

14 V. Bulatov, L. Feller, Y. Yasman and I. Schechter, Instrum. Sci. Technol., 2008, 36, 235-244.

15 E. Yakubu, B. Li, Y. Duan and S. Yang, Biomed. Opt. Express, 2018, 9, 6009.

16 N. E. Pretorius, A. Power, M. Tennant, A. Forrest and D. Cozzolino, Appl. Spectrosc. Rev., 2020, 55, 105-127.

17 S. Yang, B. Li, A. Akkus, O. Akkus and L. Lang, Analyst, 2014, 139, 3107-3114.

18 A. C. Ko, M. Hewko, M. G. Sowa, C. C. Dong, B. Cleghorn and L.-P. Choo-Smith, Opt. Express, 2008, 16, 6274.

19 J. Kekkonen, M. A. J. Finnilä, J. Heikkilä, V. Anttonen and I. Nissinen, Analyst, 2019, 144, 6089-6097.

20 A. I. Ismail, N. B. Pitts, M. Tellez and Authors of the International Caries Classification and Management System (ICCMS), BMC Oral Health, 2015, 15, S9.

21 M. D. Morris and G. S. Mandair, Clin. Orthop. Relat. Res., 2011, 469, 2160-2169.

22 S. Gamsjaeger, R. Mendelsohn, A. L. Boskey, S. GourionArsiquaud, K. Klaushofer and E. P. Paschalis, Curr. Osteoporos. Rep., 2014, 12, 454-464.

23 S. R. Goodyear, I. R. Gibson, J. M. S. Skakle, R. P. K. Wells and R. M. Aspden, Bone, 2009, 44, 899-907.

24 F. A. Shah, A. Snis, A. Matic, P. Thomsen and A. Palmquist, Acta Biomater., 2016, 30, 357-367. 
25 O. Akkus, F. Adar and M. B. Schaffler, Bone, 2004, 34, 443453.

26 T. Buchwald and Z. Buchwald, Analyst, 2019, 144, 14091419.

27 C. Xu, R. Reed, J. P. Gorski, Y. Wang and M. P. Walker, J. Mater. Sci., 2012, 47, 8035-8043.

28 F. Yun, M. V. Swain, H. Chen, J. Cairney, J. Qu, G. Sha, H. Liu, S. P. Ringer, Y. Han, L. Liu, X. Zhang and R. Zheng, Biomaterials, 2020, 235, 119748.

29 P. Seredin, D. Goloshchapov, T. Prutskij and Y. Ippolitov, PLoS One, 2015, 10, 1-11.
30 E. Wentrup-byrne, C. A. Armstrong, R. S. Armstrong and B. M. Collins, J. Raman Spectrosc., 1997, 28, 151-158.

31 E. Klimuszko, K. Orywal, T. Sierpinska, J. Sidun and M. Golebiewska, Odontology, 2018, 106, 369-376.

32 A. Surdacka, T. Matthews-Brzozowska, K. Jóźwiak and B. Stachecki, Czas. Stomatol., 1990, 43, 192-198.

33 C. Robinson, J. Kirkham, S. J. Brookes, W. A. Bonass and R. C. Shore, Int. J. Dev. Biol., 1995, 39, 145-152.

34 J. Kekkonen and I. Nissinen, 2019 IEEE Nord. Circuits Syst. Conf. NORCAS 2019 NORCHIP Int. Symp. Syst. SoC 2019 Proc., 2019, 14-18. 